\title{
Survival of Trichomonas vaginalis in human semen
}

\author{
J J DALY,* J K SHERMAN, † L GREEN,* T L HOSTETLER* \\ From the Departments of *Microbiology and Immunology, and † Anatomy, University of Arkansas for Medical \\ Sciences, Little Rock, Arkansas, USA
}

SUMMARY Although exposure of Trichomonas vaginalis to human semen is of short duration, any effect that this fluid may have on the urogenital protozoon could affect its transmission, especially if only few trichomonads are present. Small numbers of parasites (about $2500 / \mathrm{ml} \mathrm{semen)} \mathrm{incubated} \mathrm{in}$ semen from different donors at $37^{\circ} \mathrm{C}$, were found to survive or grow for up to 12 hours in all samples and for up to 24 hours in most. Survival and growth of $T$ vaginalis in semen most resembled that found in Diamond's trypticase, yeast extract, and maltose (TYM) medium without serum supplement, rather than in complete TYM medium and phosphate buffered saline. Contrary to previous reports, semen did not inhibit the survival of $T$ vaginalis, and the presence of trichomonads did not alter motility or numbers of spermatozoa up to 24 hours. The data suggest that semen provides a favourable milieu for transmitting trichomonads.

The role of men in transmitting Trichomonas vaginalis to women is not understood well. Male sexual consorts are generally accepted as being the primary sources of infection in women, but the extent to which these parasites exist in men is not clear. ${ }^{1}$ Three possibilities are usually suggested; (1) that $T$ vaginalis is only transient in men, (2) that the organism usually exists in such small numbers that it is difficult to detect and the host is symptomless, and (3) that in a few men certain symptomatology can occur, for reasons not yet clear, accompanied by a detectable number of parasites. ${ }^{23}$ Whatever the case, the vehicle for transmission from a man to an uninfected woman must be semen. For the trichomonads in semen to infect successfully they must remain alive in this transfer milieu long enough to establish themselves in a new host environment. Trichomonads have only a short exposure time to semen itself, from ejaculation to cervical transfer. After ejaculation, however, semen forms part of the material in which the parasites will begin to establish themselves in the vaginal vault. Inhibitory activity by semen could reduce the infectivity of trichomonads, especially if only few are present. The effects of semen could also play an important part in the survival of $T$ vaginalis in fresh or frozen (cryopreserved) semen used for artificial insemination.

Address for reprints: Dr J J Daly, Department of Microbiology and Immunology, University of Arkansas for Medical Sciences, 4301 West Markham, Little Rock, Arkansas, USA

Accepted for publication 14 November 1988
This study set out to assess the effect of semen on the ability of $T$ vaginalis to survive, and thus whether semen contributes to or inhibits successful transmission of trichomonads.

Patients, materials, and methods

\section{CULTURES}

Isolates of $T$ vaginalis used in these experiments were obtained from patients at the University Hospital, Little Rock, Organisms were maintained in Diamond's trypticase, yeast extract, and maltose (TYM) ${ }^{4}$ medium supplemented with $5 \%$ inactivated human serum, incubated at $37^{\circ} \mathrm{C}$, and subcultured every 48 hours.

\section{HUMAN SEMEN}

Human semen was obtained from donors selected through the University Semen Cryobank. All but one donor, who was 52, were in their 20 s and were students. All semen samples were used fresh with no cryoprotectant added.

\section{EFFECT OF HUMAN SEMEN ON SUR VIVAL OF TVAGINALIS}

\section{Inoculation and incubation}

Late $\log$ and early stationary phase cultures of $T$ vaginalis were centrifuged and washed twice with phosphate buffered saline (PBS) at pH 7.2. Cells were resuspended in PBS, the suspension was adjusted to 40 to 100 trichomonads/ $\mu 1$ PBS, and $10 \mu$ l suspension 
were added to $0.2 \mathrm{ml}$ aliquots of semen in $2 \mathrm{ml}$ volume test tubes. For anaerobic incubation, these tubes were placed unstoppered in Gas Pak jars and carbon dioxide, and hydrogen generators were employed; the jars were then closed and incubated at $37^{\circ} \mathrm{C}$. For aerobic incubation, stoppered tubes were incubated directly in a $37^{\circ} \mathrm{C}$ incubator. In all experiments, tubes of inoculated semen were removed from incubation after 6,12 , and 24 hours, and the contents were gently stirred with a bacteriology inoculating loop. Four samples of $10 \mu \mathrm{l}$ each were removed and inoculated into modified TYM medium previously maintained as liquid at $40^{\circ} \mathrm{C}$. The modified TYM medium used contained $0.45 \%$ Noble agar, $5 \%$ inactivated human serum, and antibiotics as described by Ivey. ${ }^{5}$

\section{Controls}

Tubes containing $0.2 \mathrm{ml}$ complete TYM medium were used as growth controls, TYM medium minus the serum additive as incomplete growth controls, and PBS as no growth controls. These tubes were also inoculated with $10 \mu \mathrm{l}$ trichomonad suspension and were incubated and sampled in the same way as the inoculated semen.

\section{Colony counts}

The colony count technique was used in these studies to assess population viability of trichomonads inoculated into semen and control media. This allowed the detection of small numbers of trichomonads in semen as well as the use of small aliquot volumes of individual semen specimens. Small inoculum sizes also approximated to the population densities in transient conditions and in low grade infections in men. Preparation of inoculated agar plates and colony count techniques followed the methods of Ivey, ${ }^{5}$ and Matthews and Daly. ${ }^{6}$ Inoculated liquefied TYM medium was poured into plastic petri dishes, allowed to harden, placed in Gas Pak jars containing carbon dioxide and hydrogen, and incubated for four to five days at $37^{\circ} \mathrm{C}$. Incubated plates were then counted for the number of trichomonad colonies present. As different inoculum sizes were used in each experiment, the data were expressed as percentages above the initial inocula of $100 \%$.

EFFECTS OF TRICHOMONADS ON SPERMATOZOA Pairs of semen samples (semen with and semen without trichomonads) were compared by phase microscopy for spermatozoal motility and by haemocytometer counts for numbers of spermatozoa at 0,6 , 12 , and 24 hours after incubation at $37^{\circ} \mathrm{C}$.

\section{Results}

Populations of $T$ vaginalis were found not only to
Table 1 Survival or growth of Trichomonas vaginalis in human semen or trypticase, yeast extract, and maltose (TYM) medium under anaerobic (carbon dioxide and hydrogen) or aerobic conditions at $37^{\circ} \mathrm{C}$. (Figures represent mean (SE) percent of organisms (relative to initial populations of $100 \%$ ) in five experiments)

\begin{tabular}{llllll}
\hline & \multicolumn{2}{l}{ TYM medium } & & \multicolumn{2}{l}{ Semen } \\
\cline { 2 - 3 } \cline { 5 - 5 } Hours & Aerobic & Anaerobic & & Aerobic & Anaerobic \\
\hline 6 & $51(63)$ & $94(29)$ & & $96(67)$ & $168(53)$ \\
12 & $29(49)$ & $205(109)$ & & $44(37)$ & $159(62)$ \\
24 & $41(59)$ & $450(156)$ & & $9(18)$ & $182(112)$ \\
\hline
\end{tabular}

survive in human semen at $37^{\circ} \mathrm{C}$ but also showed some growth. Five different semen specimens were examined comparing aerobic and anaerobic incubation using only the complete TYM as a control. Table 1 shows results of paired experiments on growth or survival of $T$ vaginalis in semen or complete medium incubated under aerobic compared with anaerobic conditions. Growth or survival were poorer in semen and medium under aerobic conditions than under anaerobiosis. Four other semen samples were examined for survival or growth under anaerobic conditions only, and we used complete TYM medium, TYM without serum, and PBS as control media. Table 2 shows results of paired experiments using the three growth controls. Few organisms survived in PBS after incubation for six hours. Growth in semen was most closely comparable with that in incomplete TYM medium. Although the trend at 24 hours was towards greater growth in semen, the difference was not significant $(310 \% v 116 \%)$. As expected, growth was excellent in the complete medium, but the difference between it and in semen was evident only at 24 hours $(820 \% v 310 \%, \mathrm{p}=0.005)$. Combining the data from all nine paired semen samples incubated anaerobically, survival of $T$ vaginalis at 24 hours ranged from $2 \%$ to $535 \%$ of the initial inoculum with a mean (SE) of $251(70) \%$.

No differences were found in the degree and quality of spermatozoal motility in the presence or absence of trichomonads. The means of double blind triplicate

Table 2 Survival or growth of Trichomonas vaginalis in human semen, complete trypticase, yeast extract, and maltose (TYM) growth medium, incomplete medium (TYM minus serum), and phosphate buffered saline (PBS) incubated under anaerobic (carbon dioxide and hydrogen) conditions at $37^{\circ} \mathrm{C}$. (Figures represent mean (SE) percent of organisms (relative to initial populations of $100 \%$ ) in four experiments)

\begin{tabular}{lccc}
\hline Medium & 6 hours & 12 hours & \multicolumn{1}{c}{24 hours } \\
\hline PBS & $8(16)$ & $6(11)$ & $0 \cdot 3(0 \cdot 5)$ \\
Incomplete TYM & $141(81)$ & $171(98)$ & $116(214)$ \\
Semen & $195(117)$ & $213(106)$ & $310(175)$ \\
Complete TYM & $221(127)$ & $253(131)$ & $820(200)$ \\
\hline
\end{tabular}


motility readings in aliquots of semen with $v$ without trichomonads were $51 v 52 \%$ initially, $44 v 43 \%$ after 6 hours, $22 v 21 \%$ after 12 hours, and $2 v 2 \%$ after 24 hours. The degree and quality of motility were indistinguishable between media at the same observation periods, apart from heightened spermatozoal agglutination initially in one sample with parasites. Spermatozoal microscopy rarely showed motile trichomonads initially (because only small numbers were present), but they were easily found in the 24 hour samples. Duplicate and mean measurements of the total numbers or concentration (millions $/ \mathrm{ml}$ ) of spermatozoa during incubation for $\mathbf{2 4}$ hours showed no change. We found no evidence, therefore, for phagocytosis of spermatozoa by trichomonads in incubated infected semen, in contrast to the spermatozoal phagocytosis by trichomonads reported for vaginal secretions. $^{7}$

\section{Discussion}

Published reports indicate that certain components of seminal fluid may inhibit the survival of $T$ vaginalis. Canine and human prostatic fluids have been found to inhibit trichomonal survival. ${ }^{89}$ This was related to the presence of zinc in other experiments in which the trichomonads were directly exposed to this ion. ${ }^{8-10}$

Previous investigators used phase microscopy to detect viable trichomonads in microtitre plates, but failed to find $T$ vaginalis surviving in seminal fluid after incubation for six hours at $30^{\circ} \mathrm{C}^{8}$ In contrast to these findings, we found excellent survival of $T$ vaginalis in semen from different donors after incubation for up to 24 hours at $37^{\circ} \mathrm{C}$. The failure of other workers to find viable $T$ vaginalis in seminal fluid incubated for six hours may have been due to the large size of their initial inoculum (40 000 trichomonads $/ \mathrm{ml}$ seminal fluid) or the different incubation temperatures, or both. In the study reported here the colony formation technique allowed us to use a small inoculum (about 2500 cells $/ \mathrm{ml}$ semen), which would more approximate to populations of trichomonads seen in infected men.

As the data presented herein indicated that semen acts as an incomplete medium, it follows that the limiting factor(s) for growth would shorten the growth curve considerably in the presence of a large inoculum. Using colony counts as a measurement of viabilty, the stationary phase of $T$ vaginalis in growth medium has been shown to be short and the death or decline phase pronounced. ${ }^{11}$ The trichomonads in seminal fluid were possibly too far into the death phase to remain viable or to retain active motilty. We are currently examining the effect of different inoculum sizes on trichomonad survival in semen.
A factor other than zinc may also be responsible for the inability of trichomonads to thrive in the urogenital tracts of men. ${ }^{810}$ The work published here suggests that one factor could be nutritional, as the growth response indicated that semen was an incomplete medium for $T$ vaginalis. Poor survival in prostatic fluid may be due to the lack of carbohydrate in the form of fructose, which is found in seminal fluid. ${ }^{12}$ Another possibility (yet to be tested) to explain the results in the present report, is that the presence of spermatozoa may enhance trichomonad survival. Our finding of no effect by trichomonads on spermatozoal motility does not support a report by Tuttle et al that a striking decrease in motility occurs during incubation with these parasites at $37^{\circ} \mathrm{C}$ for six hours. ${ }^{13}$ The high concentration of trichomonads that they used (about five times our "normal" numbers) were possibly the causative variable.

In conclusion, the survival data in the present report support the view that semen can serve as a good vehicle for transmiting trichomonads.

We thank Joy Thompson for assistance in assessing spermatozoal motility and numbers.

\section{References}

1 Krieger JN. Urologic aspects of trichomoniasis. Investigative Urology 1981;18:411-7.

2 Gardner WA, Culberson DE, Bennett BD. Trichomonas vaginalis in the prostate gland. Arch Pathol Lab Med 1987;110:430-2.

3 Latif AS, Mason PR, Marowa E. Urethral trichomoniasis in men. Sex Transm Dis 1987;14:9-11.

4 Diamond LS. The establishment of various trichomonads of animals and man in axenic cultures. J Parasitol 1957;43:488-90.

5 Ivey MH. Growth characteristics of clones of Trichomonas vaginalis in solid medium. $J$ Parasitol 1961;47:539-44.

6 Matthews RS, Daly JJ. Trichomonas gallinae: use of solid medium to test survival under various environmental conditions. Exp Parasitol 1974;36:288-98.

7 Kolesoff, AP. Akusherstovo i Ginekologiya 1950; No 6:46. Cited in: Bernfeld WK. A note on Trichomonas vaginalis and seminal fluid. British Journal of Venereal Diseases 1972;48:144-5.

8 Langley JG, Goldsmid JM, Davies N. Venereal trichomoniasis: role of men. Genitourin Med 1987;63:264-7.

9 Krieger JN, Rein MF. Canine prostatic secretions kill Trichomonas vaginalis. Infect Immun 1982;37:77-81.

10 Krieger JN, Rein MF. Zinc sensitivity of Trichomonas vaginalis: in vitro studies and clinical implications. J Infect Dis 1982; 146:341-5.

11 Daly JJ, Baker ML, Burton SB. The sensitivity of Trichomonas vaginalis and Trichomonas gallinae to ultraviolet radiation. Photochem Photobiol 1981;33:191-6.

12 Eliasson R, Lindholmer C. Functions of male accessory genital organs. In: Hafez ESE, ed. Human semen and fertility regulation in man. St. Louis: CV Mosby, 1976;44-50.

13 Tuttle JP Jr, Holbrook TW, Derrick FC. Interference of human spermatozoal motility by Trichomonas vaginalis. J Urol 1977; 118:1024-5. 